\title{
Abnormal spontaneous regional brain activity in primary insomnia: a resting-state functional magnetic resonance imaging study
}

This article was published in the following Dove Press journal:

Neuropsychiatric Disease and Treatment

14 June 2016

Number of times this article has been viewed

\author{
Chao $\mathrm{Li}^{\prime}$ \\ Xiaofen $\mathrm{Ma}^{2}$ \\ Mengshi Dong² \\ YiYin' \\ Kelei Hua' \\ Meng $\mathrm{Li}^{2}$ \\ Changhong $\mathrm{Li}^{2}$ \\ Wenfeng Zhan ${ }^{2}$ \\ Cheng $\mathrm{Li}^{2,3}$ \\ Guihua Jiang ${ }^{2}$ \\ 'Department of Medical Imaging, \\ The Affiliated Guangdong No 2 \\ Provincial People's Hospital of \\ Southern Medical University, \\ The Third Clinical Medical College \\ of Southern Medical University, \\ ${ }^{2}$ Department of Medical Imaging, \\ ${ }^{3}$ Department of Renal Transplantation, \\ The Affiliated Guangdong No 2 \\ Provincial People's Hospital of \\ Southern Medical University, \\ Guangzhou, People's Republic \\ of China
}

Correspondence: Guihua Jiang

Department of Medical Imaging,

The Affiliated Guangdong No 2 Provincial

People's Hospital of Southern Medical

University, Guangzhou 510317, People's

Republic of China

Tel/fax +86 2089168071

Email jiangguihual970@।63.com

Cheng Li

Department of Renal Transplantation,

The Affiliated Guangdong No 2 Provincial

People's Hospital of Southern Medical

University, Guangzhou 510317, People's

Republic of China

Tel/fax +8620 I392422 8992

Email I82838li@I63.com
Objective: Investigating functional specialization is crucial for a complete understanding of the neural mechanisms of primary insomnia (PI). Resting-state functional magnetic resonance imaging (fMRI) is a useful tool to explore the functional specialization of PI. However, only a few studies have focused on the functional specialization of PI using resting-state fMRI and results of these studies were far from consistent. Thus, the current study aimed to investigate functional specialization of PI using resting-state fMRI with amplitude of low frequency fluctuations (ALFFs) algorithm.

Methods: In this study, 55 PI patients and 44 healthy controls were included. ALFF values were compared between the two groups using two-sample $t$-test. The relationship of abnormal ALFF values with clinical characteristics and duration of insomnia was investigated using Pearson's correlation analysis.

Results: PI patients showed lower ALFF values in the left orbitofrontal cortex/inferior frontal gyrus, right middle frontal gyrus, left inferior parietal lobule, and bilateral cerebellum posterior lobes, while higher ALFF values in the right middle/inferior temporal that extended to the right occipital lobe. In addition, we found that the duration of PI negatively correlated with ALFF values in the left orbitofrontal cortex/inferior frontal gyrus, and the Pittsburgh Sleep Quality Index score negatively correlated with ALFF values in the left inferior parietal lobule.

Conclusion: The present study added information to limited studies on functional specialization and provided evidence for hyperarousal hypothesis in PI.

Keywords: primary insomnia, amplitude of low frequency fluctuations, resting-state fMRI, spontaneous brain activity

\section{Introduction}

Primary insomnia (PI) is defined as the presence of insomnia symptoms unrelated to any other medical, physical, or psychiatric disorder. ${ }^{1}$ Nearly one-third of adults suffer from insomnia in any given year; if acute insomnia is considered, the diagnostic prevalence rises to almost $50 \%$ of the population. ${ }^{2,3}$ Patients with insomnia often suffer from cognitive impairment and even mortality. ${ }^{4}$ Although insomnia seriously affect people's physical and mental health, the neurobiological mechanisms underlying PI are not clear.

Both task-based and resting-state functional magnetic resonance imaging (fMRI) have emerged as a useful tool to explore the neuromechanism of $\mathrm{PI}^{5-12}$ or sleep deprivation. ${ }^{13} \mathrm{fMRI}$ is a novel noninvasive imaging technique for measuring spontaneous brain activity ${ }^{14,15}$ and more economical to implement than positron emission tomography and single photon emission computed tomography in clinical studies. ${ }^{16}$ Using resting-state fMRI with amplitude of low frequency fluctuations (ALFFs) algorithm, 
Gao et $\mathrm{al}^{13}$ found that 24 hours sleep deprivation led prominently decreased ALFF values in the right inferior parietal lobule, bilateral orbitofrontal cortex, and dorsolateral prefrontal cortex, while increased ALFF values in the visual cortex, left sensorimotor cortex, and fusiform gyrus. Using restingstate fMRI with functional connectivity method, Huang et $\mathrm{al}^{5}$ explored decreased temporal connectivity between the amygdala and striatum, insula, and thalamus, which is mainly an emotion circuit, which provided further evidence for hyperreactivity to perceived threat ${ }^{17}$ in PI patients. Li et $\mathrm{al}^{6}$ examined the temporal correlation between the superior parietal lobe and whole-brain regions in PI patients. They found that PI patients showed abnormal functional connectivity between the parietal and prefrontal cortices and the anterior cingulate gyrus, which they thought may be due to the impairment of spatial and verbal working memory.

However, to our best knowledge, only a few studies ${ }^{7,8,18}$ have explored the spontaneous regional brain activity or functional specialization in PI using resting-state fMRI. Paying attention to functional specialization is crucial for detecting precisely which region of the brain was abnormal and completely understanding of the neuromechanisms in PI. Unfortunately, results of these studies focusing on function specialization were far from consistent. For example, Dai et al ${ }^{18}$ found that compared with good sleepers, PI patients showed higher regional homogeneity (ReHo) in the left fusiform gyrus and lower ReHo in the bilateral cingulate gyrus and right cerebellum anterior lobe. In contrast to the study of Dai et al, Wang et $\mathrm{l}^{8}$ reported increased ReHo in the left insula, right anterior cingulate gyrus, bilateral precentral gyrus, and left cuneus, as well as decreased ReHo in the right middle cingulate cortex and left fusiform. Recently, Dai et $\mathrm{al}^{7}$ found that both PI female and male patients showed higher ALFF in the temporal lobe and occipital lobe. They also found female PI patients had lower ALFF in the bilateral cerebellum posterior lobe, left dorsolateral prefrontal cortex, and bilateral limbic lobe; however, male PI patients showed lower ALFF in the left occipital gyrus. The question remains as whether and how can the PI affect the regional spontaneous activity pattern of human brain.

Thus, the objective of this study was to explore the spontaneous regional brain activity of PI. We selected restingstate $\mathrm{fMRI}$ with an ALFF algorithm as an index to measure spontaneous regional brain activity. Compared with other algorithms, such as a ReHo and functional connectivity, ALFF can directly reflect the intensity or amplitude of spontaneous regional brain activity. ${ }^{19}$ Besides, ALFF method has favorable test-retest reliability. ${ }^{20}$ ALFF has been successfully applied in many psychiatric and neurological diseases, such as major depressive disorder, ${ }^{21}$ schizophrenia, ${ }^{22}$ and attention deficit hyperactivity disorder. ${ }^{19}$ We hypothesized that PI patients may have different ALFF values in some brain regions, such as lower ALFF values in the prefrontal cortex based on previous consistent findings ${ }^{11,23-26}$ and higher ALFF values in auditory and visual cortices based on hyperarousal hypothesis ${ }^{27,28}$ and previous findings. ${ }^{7}$ We also hypothesized that ALFF values in abnormal regions may be correlated with some clinical characteristics and duration of insomnia.

\section{Methods \\ Subjects}

The study was approved by the local ethics committee of the Institute of Mental Health at the Guangdong No 2 Provincial People's Hospital. All subjects signed written informed consent prior to inclusion. From April 2010 to April 2016, 55 patients with PI ([mean age: $39 \pm 10$ years], including 24 men [mean age: $39 \pm 10$ years] and 31 women [mean age: $39 \pm 10$ years]) were included. Patients who met the following criteria were included in the study: 1) conformity to the definition of PI by the Diagnostic and Statistical Manual of Mental Disorders, 4th Edition; 2) insomnia lasting $\geq 1$ months, with a complaint of difficulty falling asleep, maintaining sleep, or early awakening; 3 ) no other sleep disorders (such as hypersomnia, parasomnia, or sleep-related movement disorder) or other psychiatric disorders determined by a semistandardized psychiatric and sleep-related interview conducted by an experienced psychiatrist; 4) insomnia dose not due to the effects of medications/substance abuse, such as caffeine or nicotine or alcohol; 5) right-hand dominance; 6) no serious organic disease; 7) no foreign implants in the body; 8) age 25-60 years; 9) no abnormal signal as verified by conventional T1- or T2-fluid-attenuated inversion recovery (FLAIR) MRI; and 10) head motion $<1.5 \mathrm{~mm}$ or $1.5^{\circ}$ during MRI.

Forty-four age-, sex-, and education-level-matched healthy control subjects (mean age: $39 \pm 9$ years), including 11 men (mean age: $38 \pm 6$ years) and 33 women (mean age: $40 \pm 10$ years) were recruited from the local community. All control subjects met the following criteria: 1) good sleeping habits and good sleep onset and/or maintenance; 2) no history of swing shifts, shift work, or sleep complaints; 3) no medications/substance abuse, such as caffeine or nicotine or alcohol; and 4) fulfillment of criteria 5-10 above for the PI patients.

\section{Questionnaires}

All volunteers were asked to complete a number of questionnaires, including the Pittsburgh Sleep Quality Index (PSQI), the Insomnia Severity Index (ISI), the Self-rating Anxiety 
Scale (SAS), and the Self-rating Depression Scale (SDS) to evaluate the sleep situation and the mental status of the PI patients.

\section{MRI data acquisition}

Participants completed a 10-minute resting-state fMRI scan (240 volumes) acquired on a Philips Achieva 1.5T Nova dual MR scanner. Head motion was restricted by a belt and foam pads. During resting state fMRI scanning, subjects were instructed to close their eyes, but not to fall asleep and not to think of anything particularly. The restingstate fMRI images were obtained using a gradient-echo planar imaging sequence (interleaved scanning, repetition time $/$ echo time $=2,500 \mathrm{~ms} / 50 \mathrm{~ms}$, matrix $=64 \times 64$, field of view $=224 \times 224 \mathrm{~mm}$, flip angle $=90^{\circ}$, section thickness $=4 \mathrm{~mm}$, gap $=0.8 \mathrm{~mm}, 27$ sections covering the whole brain).

\section{Data processing and ALFF calculations}

Data Processing Assistant for Resting-State Functional MR Imaging toolbox (http://www.restfmri.net/forum/DPARSF $)^{29}$ was used to postprocess the imaging data. Functional images at the first ten volumes were discarded so that magnetization reached steady state and subjects adapted to the MRI scanning noise. The slice timing and realignment for head motion correction and spatial normalization were conducted according to the standard Montreal Neurologic Institute template, resampled into a voxel size of $3 \times 3 \times 3 \mathrm{~mm}^{3}$. We then smoothed these images by convolution with an isotropic Gaussian kernel (full width at half maximum, $6 \mathrm{~mm}$ ). To reduce the effects of low-frequency drift and high-frequency noise, the smoothed imaging data were processed to remove linear trends and be filtered temporally (band pass, $0.01-0.08 \mathrm{~Hz}$ ). Three patients and three control subjects who showed head motion of $>1.5 \mathrm{~mm}$ or $1.5^{\circ}$ during image acquisition were excluded in subsequent analysis. ALFF was calculated using the toolbox previously described. ${ }^{19}$ Briefly, the time series for each given voxel was first converted to a frequency domain using a Fast Fourier Transform. The square root of the power spectrum was computed and averaged throughout $0.01-0.08 \mathrm{~Hz}$ at each voxel. This averaged square root was termed the ALFF for each voxel. For standardization purposes, the ALFF of each voxel was divided by the global mean ALFF value of each individual. The standardized ALFF value for each given voxel reflected the degree of its raw ALFF value relative to the average ALFF value of the global brain.

\section{Statistical analysis}

The SPSS Version 20.0 (IBM Corporation, Armonk, NY, USA) was used to analyze demographic data and clinical characteristics. Statistical parametric mapping software (SPM8, http://www.fil.ion.ucl.ac.uk/spm) was used to analyze fMRI data in a voxel-by-voxel fashion. To investigate ALFF difference between the two groups, we used a twosample $t$-test on the individual ALFF maps in a voxel-byvoxel fashion, with sex, age, and education level imported as covariates. A corrected significance level of individual voxel $P<0.01$ and the contiguous cluster volume $\geq 540 \mathrm{~mm}^{3}$ were used to determine the statistical significance (corrected for multiple comparisons with AlphaSim corrected threshold of cluster $P<0.05$, http://afni.nimh.nih.gov/afni/). Sex distribution among the two groups was evaluated using the chi-square test. Differences in age, ISI, PSQI, SAS, and SDS scores between the two groups were calculated with two-sample $t$-test. Differences in education level between the two groups were compared with Mann-Whitney $U$ statistic.

To identify the association between the ALFF values and the PSQI, ISI, SAS, and SDS scores and duration of PI, the mean ALFF values for all voxels in the significant areas were extracted separately using the Resting-State fMRI Data Analysis Toolkit (http://resting-fmri.sourceforge.net). Then, Pearson's correlation analysis was performed between the mean ALFF values in significantly different areas and the PSQI, ISI, SAS, and SDS scores and duration of PI in SPSS $20.0(P<0.05)$.

\section{Results}

\section{Demographic and clinical characteristics}

Table 1 provides demographic and clinical characteristics for PI patients and controls. There were no differences in sex, age, and education level between the patient and control groups $(P>0.05)$. PI patients had higher PSQI, ISI, SAS, and SDS scores than controls $(P<0.05)$.

Table I Characteristics of subjects

\begin{tabular}{llll}
\hline Parameter & PI (55) & HC (44) & P-value \\
\hline Sex* & & & $0.086^{\mathrm{a}}$ \\
$\quad$ Male & 24 & $\mathrm{II}$ & \\
$\quad$ Female & 31 & 33 & \\
Age (year) & $39.18 \pm 10.34$ & $39.91 \pm 9.43$ & $0.72^{\mathrm{b}}$ \\
Education (year) & $7.47 \pm 3.58$ & $8.30 \pm 4.21$ & $0.24^{\mathrm{c}}$ \\
Insomnia duration (month) & $46.04 \pm 29.63$ & $\mathrm{~N} / \mathrm{A}$ & $\mathrm{N} / \mathrm{A}$ \\
PSQI & $12.51 \pm 3.25$ & $5.93 \pm 2.27$ & $0.00^{\mathrm{b}}$ \\
ISI & $19.69 \pm 3.28$ & $5.36 \pm 1.14$ & $0.00^{\mathrm{b}}$ \\
SAS & $52.05 \pm 10.73$ & $42.33 \pm 5.21$ & $0.00^{\mathrm{b}}$ \\
SDS & $54.62 \pm 8.7 \mathrm{I}$ & $42.32 \pm 6.35$ & $0.00^{\mathrm{b}}$ \\
\hline
\end{tabular}

Notes: Unless otherwise indicated, data are mean \pm standard deviation. $*$ Data are number of patients or control subjects. ${ }^{a}$ The $P$-values were obtained by using the chisquare test. 'The $P$-values were obtained by using a two-sample $t$-test. ${ }^{\text {'T }}$ The $P$-values were obtained by using Mann-Whitney $U$ statistic.

Abbreviations: ISI, Insomnia Severity Index; PI, primary insomnia; PSQI, Pittsburgh Sleep Quality Index; SAS, Self-rating Anxiety Scale; SDS, Self-rating Depression Scale; N/A, not applicable; HC, healthy controls. 
Table 2 Brain regions showing significant differences of ALFF in PI patients compared with healthy controls

\begin{tabular}{|c|c|c|c|c|c|c|}
\hline \multirow[t]{2}{*}{ Location } & \multirow[t]{2}{*}{ Voxel size } & \multicolumn{3}{|c|}{ MNI coordinates } & \multirow[t]{2}{*}{$T$-value } & \multirow[t]{2}{*}{ Brodmann area } \\
\hline & & $\bar{x}$ & $Y$ & $Z$ & & \\
\hline Right cerebellum & 3,213 & 15 & -60 & -48 & -4.10 & N/A \\
\hline Left cerebellum & $\mathrm{I}, 107$ & -15 & -57 & -48 & -3.60 & N/A \\
\hline $\begin{array}{l}\text { Left orbitofrontal } \\
\text { cortex/inferior frontal gyrus }\end{array}$ & 729 & -21 & 33 & -21 & -3.62 & $\mathrm{II}, 47$ \\
\hline Right middle frontal gyrus & 594 & 33 & 3 & 48 & -3.95 & 6,9 \\
\hline Left inferior parietal lobule & 648 & -42 & -39 & 33 & -4.08 & 40 \\
\hline $\begin{array}{l}\text { Right middle/inferior temporal } \\
\text { gyrus, occipital lobe }\end{array}$ & 567 & 57 & -63 & -3 & 4.32 & 19,37 \\
\hline
\end{tabular}

Abbreviations: ALFF, amplitude of low frequency fluctuations; PI, primary insomnia; MNI, Montreal Neurological Institute; N/A, not applicable.

\section{Regional brain ALFF values comparison}

We found significantly abnormal spontaneous regional brain activity in six clusters in the PI patients compared with the control group (Table 2 and Figure 1). PI patients displayed lower ALFF values in the left orbitofrontal cortex/inferior frontal gyrus, right middle frontal gyrus, left inferior parietal lobule, and bilateral cerebellum posterior lobes, while higher ALFF values in the right middle/inferior temporal that extended to the right occipital lobe.

\section{Correlation between ALFF values and PSQI, ISI, SAS, and SDS scores and duration of $\mathrm{PI}$}

Figure 2 shows the results of correlation analysis. The duration of PI negatively correlated with ALFF values in the left orbitofrontal cortex/inferior frontal gyrus $(r=-0.492$, $P=0.001)$. The PSQI score negatively correlated with ALFF values in the left inferior parietal lobule $(r=-0.324$, $P=0.016$ ). Current study did not detect significant correlation between ALFF values in abnormal regions and ISI, SAS, and SDS scores.

\section{Discussion}

Using resting-state fMRI with ALFF algorithm, we explored differences in spontaneous regional brain activity between a group of PI patients and a group of healthy controls. Statistical analysis indicated that compared with controls, the PI patients showed significantly lower ALFF values in some brain regions associated with alertness, attention, and higher-order cognitive processes, while PI patients showed significantly higher ALFF values in auditory-related and vision-related regions. Additionally, the ALFF values of some regions showed a significant correlation with the clinical features of PI patients. Current study may play an important role in precisely figuring out the abnormal brain regions and provide evidence for hyperarousal hypothesis.
Consistent with previous studies, we detected lower ALFF values in the prefrontal lobe, including the left orbitofrontal cortex/inferior frontal gyrus and right middle frontal gyrus (Table 2 and Figure 1). ${ }^{11,13,23-26,30}$ The prefrontal cortex is thought to be involved in alertness, attention, and higher-order cognitive processes ${ }^{26}$ Using task fMRI, Altena et $\mathrm{al}^{11}$ found that chronic insomnia patients showed hypoactivation of the medial and inferior prefrontal cortical areas during the performance of a category and a letter fluency task, which recovered after sleep therapy. This findings suggested that chronic insomnia can damage prefrontal cortex function. Using $\left[{ }^{18} \mathrm{~F}\right]$-fluorodeoxyglucose positron emission tomography, Nofzinger et a ${ }^{23}$ also found PI patients showed reduced relative metabolism in the prefrontal cortex while awake, and they speculated that daytime fatigue may reflect decreased activity in the prefrontal cortex resulting from inefficient sleep. Brain morphological studies also demonstrated structural abnormalities in the prefrontal cortex in PI patients. Using voxel-based morphometry, Joo et $\mathrm{al}^{30}$ found that PI patients displayed significantly reduced gray matter concentrations in dorsolateral prefrontal and pericentral cortices, superior temporal gyrus, and cerebellum and decreased gray matter volumes in medial frontal and middle temporal gyri compared with controls. Evidences from sleep deprivation also support our findings. For example, a recent resting-state fMRI study with ALFF algorithm found that healthy people who underwent sleep deprivation showed decreased ALFF in the right inferior parietal lobule, bilateral orbitofrontal cortex, and dorsolateral prefrontal cortex. ${ }^{13}$

Notably, the most recent animal study performed by Bellesi et $\mathrm{al}^{25}$ further deepened our understanding of hypoactivation or hypoperfusion in prefrontal cortex in PI and individuals experienced sleep deprivation. They found that the noradrenaline level in prefrontal cortex declined after extended prolonged wakefulness. This result suggests that locus coeruleus neurons, the primary source of 


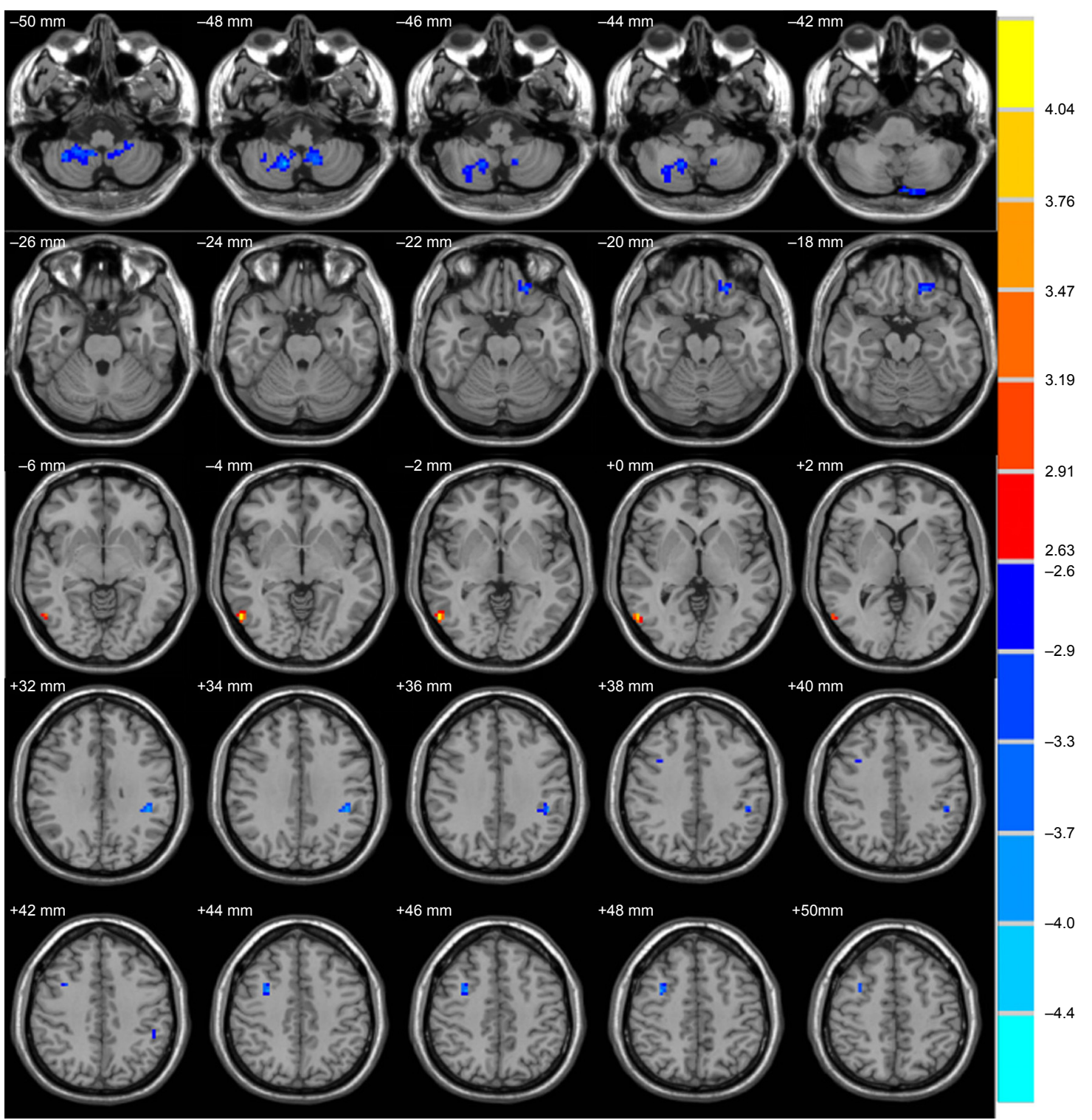

Figure I Clusters showing statistically significant different ALFF values in patients with PI compared with the controls $(P<0.0$ I, corrected). Numbers indicate $z$ slice and are displayed in MNI coordinates.

Abbreviations: ALFF, amplitude of low frequency fluctuations; MNI, Montreal Neurological Institute; PI, primary insomnia.

noradrenaline in brain, projecting to prefrontal cortex are not able to maintain noradrenaline release for long periods of time. Based on previous studies mentioned above, we speculate that the lower ALFF values in the prefrontal cortex may represent locus coeruleus neural fatigue. ${ }^{24}$ In other words, the lower ALFF values in the prefrontal cortex may be caused by extended hyperactivity in these areas in view of the hyperarousal hypothesis..$^{27,28}$ Our speculation was also supported by our correlation analysis that the duration of PI negatively correlated with ALFF values in the left orbitofrontal cortex/inferior frontal gyrus. Thus, the longer the duration of PI, the lower would be the spontaneous activity in the prefrontal cortex.
We also observed significantly decreased ALFF values in the left inferior parietal lobule, which is consistent with previous studies. ${ }^{12,13,31}$ Using task fMRI with working memory paradigm, Drummond et al ${ }^{12}$ found that PI patients showed reduced activation of task-related working memory regions (main effect of group) compared with controls, including bilateral inferior parietal lobes. Gao et $\mathrm{a}^{13}$ also detected lower ALFF values in the inferior parietal lobule in those who experienced sleep deprivation. Inferior parietal lobule is main node of the Default Mode Network ${ }^{32}$ characterized by more energetic metabolic and neural activity at rest. A proposed function for sleep is brain energy restoration. ${ }^{33}$ PI patients with poor sleep quality will probably disturb this 

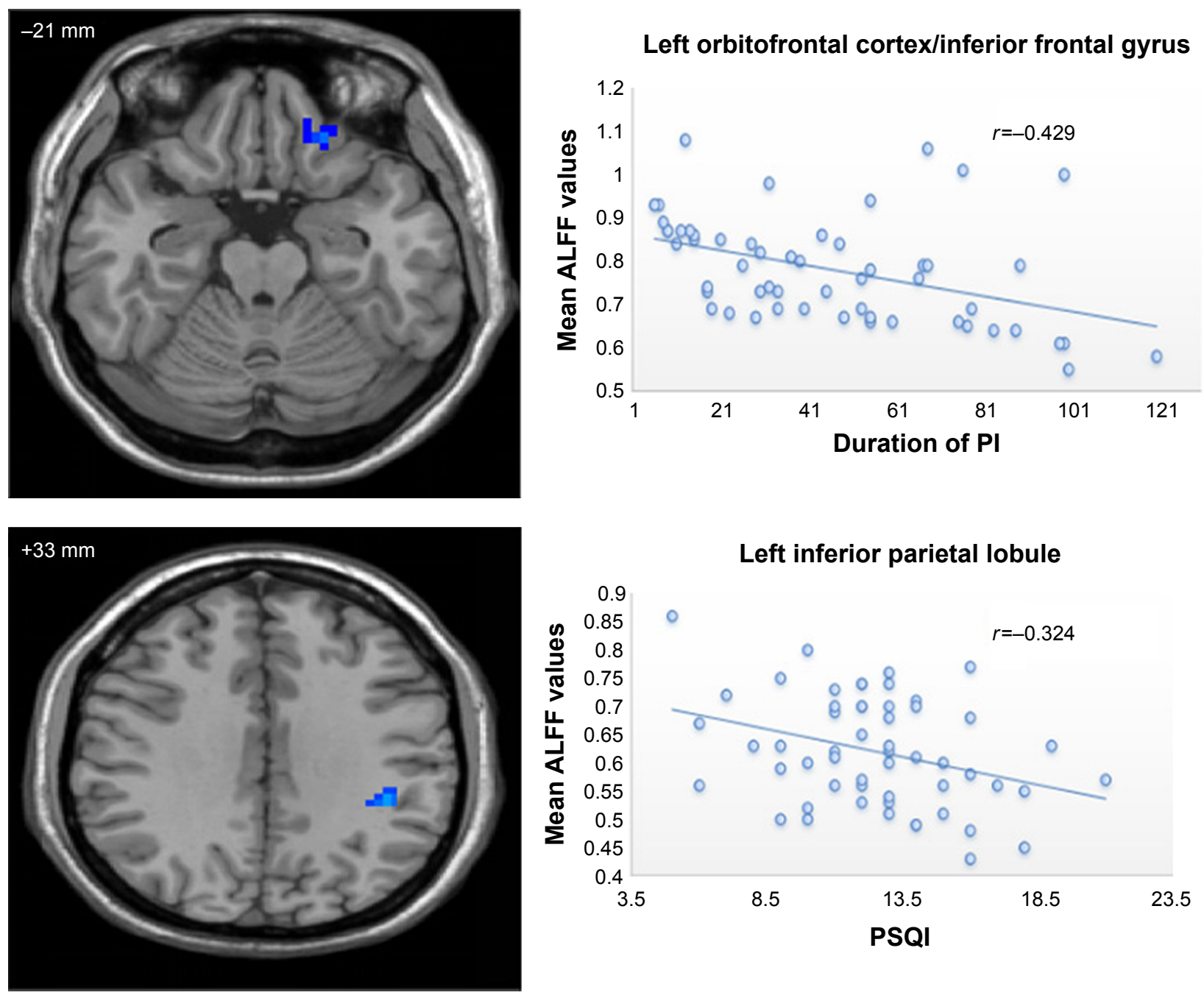

Figure 2 Scatter plot of the mean ALFF values in the left orbitofrontal cortex/inferior frontal gyrus and left inferior frontal gyrus changing with the duration of PI and PSQI, respectively.

Abbreviations: ALFF, amplitude of low frequency fluctuations; PI, primary insomnia; PSQI, Pittsburgh Sleep Quality Index.

energy restoration process. Consequently, based on previous findings, the lower ALFF values in the left inferior parietal lobule may result from disturbance of energy restoration process caused by poor sleep quality. Our proposal was supported by previous study ${ }^{31}$ and our correlation analysis. Sexton et $\mathrm{al}^{31}$ found that greater rate of atrophy in left parietal lobe in community-dwelling adults was associated with poor sleep quality represented by higher PSQI score. Current study also found that lower ALFF values in left parietal lobe was associated with poor sleep quality. Thus, both structure and function of parietal lobe are impaired by poor sleep quality.

The bilateral cerebellum posterior lobes of PI patients in our study showed decreased ALFF values. Although the traditional view is that the cerebellum is responsible for motor control, ${ }^{34}$ there is growing evidence that the cerebellum has other high order function, ${ }^{35}$ such as sensory acquisition and discrimination. ${ }^{36}$ Recently, it has been found that the cerebellum is involved in the regulation of sleep. ${ }^{37}$ For example, Joo et a $\mathrm{l}^{30}$ found that chronic PI patients had reduced gray matter concentrations in cerebellum gray matter, and Dai et $\mathrm{al}^{7}$ found that female chronic PI patients showed lower ALFF in bilateral cerebellum. Similar to above findings, we also found PI patients showed lower ALFF in bilateral cerebellum, which further proves that the cerebellum may be involved in the regulation of sleep.

The temporal lobe and occipital lobe are auditory-related and vision-related regions. Our study detected higher ALFF values in these two regions, include the fusiform gyrus (BA 37) and associative visual cortex (BA 19), in PI patients. These results support hyperarousal hypothesis. ${ }^{27}$ According to hyperarousal hypothesis, PI patients often showed hyperperfusion in cortical and subcortical structures ${ }^{23}$ and increased beta EEG activity ${ }^{28}$ in the central nerve system. Dai et $\mathrm{al}^{7}$ also 
found PI patients showed higher ALFF values in the temporal lobe and occipital lobe. Together with previous studies, our results demonstrated that PI patients had higher spontaneous brain activity in auditory-related and vision-related regions.

We acknowledge several limitations of our study. First of all, we did not explore the sex differences in ALFF values in PI patients. However, our research motivation mainly came from the fact that only a few studies have explored the spontaneous regional brain activity of PI and results of these studies were far from consistent. Second, we did not detect the restingstate fMRI data of PI patients during sleep. Future researches could investigate the ALFF of PI patients during sleep so that researchers can directly look insight resting-state brain activity during sleep. Third, the prefrontal cortex and inferior parietal lobe are the regions responsible for higher-order function, such as attention and working memory. ${ }^{35}$ However, we did not design a relevant scale to test PI patients. Fourth, this was a case-control study. A longitudinal study measuring ALFF in PI patients before and after treatment, especially cognitive behavioral therapy, is necessary to test whether ALFF can be used as a biomarker for curative effect.

\section{Conclusion}

We detected both lower and higher amplitude of spontaneous regional brain activities in PI patients. The brain regions associated with higher-order cognitive processes, alertness, attention, auditory, and vision should be paid special attention to. Current study provided evidence for hyperarousal hypothesis in PI. These results, together with limited previous studies and future studies on functional specialization in PI patients, may eventually figure out those precise regions where the spontaneous brain activities were abnormal.

\section{Acknowledgments}

The authors would like to express their appreciation to editors and two anonymous reviewers for their works and constructive advice. This work was supported by the National Natural Science Foundation of China (81471639), the Natural Science Foundation of Guangdong Province, China (2015A030313723), and the Natural Science Foundation of Guangdong Province, China (2014A030313673).

\section{Disclosure}

The authors report no conflicts of interest in this work.

\section{References}

1. Association AP. Diagnostic and Statistical Manual of Mental Disorders (DSM). Washington, DC: American Psychiatric Association. 1994: $143-147$.
2. Ohayon MM. Epidemiology of insomnia: what we know and what we still need to learn. Sleep Med Rev. 2002;6:97-111.

3. Riemann D, Nissen C, Palagini L, Otte A, Perlis ML, Spiegelhalder K. The neurobiology, investigation, and treatment of chronic insomnia. Lancet Neurol. 2015;14:547-558.

4. Vgontzas AN, Fernandez-Mendoza J, Liao D, Bixler EO. Insomnia with objective short sleep duration: the most biologically severe phenotype of the disorder. Sleep Med Rev. 2013;17:241-254.

5. Huang Z, Liang $P$, Jia $X$, et al. Abnormal amygdala connectivity in patients with primary insomnia: evidence from resting state fMRI. Eur J Radiol. 2012;81:1288-1295.

6. Li Y, Wang E, Zhang H, et al. Functional connectivity changes between parietal and prefrontal cortices in primary insomnia patients: evidence from resting-state fMRI. Eur J Med Res. 2014;19:32.

7. Dai XJ, Nie X, Liu X, et al. Gender differences in regional brain activity in patients with chronic primary insomnia: evidence from a resting-state fMRI study. J Clin Sleep Med. 2016;12(3):363-374.

8. Wang T, Li S, Jiang G, et al. Regional homogeneity changes in patients with primary insomnia. Eur Radiol. 2016;26(5):1292-1300.

9. Nie X, Shao Y, Liu SY, et al. Functional connectivity of paired default mode network subregions in primary insomnia. Neuropsychiatr Dis Treat. 2015;11:3085-3093.

10. Chen MC, Chang C, Glover GH, Gotlib IH. Increased insula coactivation with salience networks in insomnia. Biol Psychol. 2014; 97:1-8.

11. Altena E, Van Der Werf YD, Sanz-Arigita EJ, et al. Prefrontal hypoactivation and recovery in insomnia. Sleep. 2008;31:1271-1276.

12. Drummond SP, Walker M, Almklov E, Campos M, Anderson DE, Straus LD. Neural correlates of working memory performance in primary insomnia. Sleep. 2013;36:1307-1316.

13. Gao L, Bai L, Zhang Y, et al. Frequency-dependent changes of local resting oscillations in sleep-deprived brain. PLoS One. 2015;10: e120323.

14. Biswal B, Zerrin Yetkin F, Haughton VM, Hyde JS. Functional connectivity in the motor cortex of resting human brain using echo-planar MRI. Magn Reson Med. 1995;34:537-541.

15. Fox MD, Greicius M. Clinical applications of resting state functional connectivity. Front Syst Neurosci. 2010;4:19.

16. Greicius M. Resting-state functional connectivity in neuropsychiatric disorders. Curr Opin Neurol. 2008;21:424-430.

17. Baglioni C, Spiegelhalder K, Lombardo C, Riemann D. Sleep and emotions: a focus on insomnia. Sleep Med Rev. 2010;14:227-238.

18. Dai XJ, Peng DC, Gong HH, et al. Altered intrinsic regional brain spontaneous activity and subjective sleep quality in patients with chronic primary insomnia: a resting-state fMRI study. Neuropsychiatr Dis Treat. 2014;10:2163-2175.

19. Zang YF, He Y, Zhu CZ, et al. Altered baseline brain activity in children with ADHD revealed by resting-state functional MRI. Brain Dev. 2007;29:83-91.

20. Zuo XN, Di Martino A, Kelly C, et al. The oscillating brain: complex and reliable. Neuroimage. 2010;49:1432-1445.

21. Yao Z, Yan R, Wei M, Tang H, Qin J, Lu Q. Gender differences in brain activity and the relationship between brain activity and differences in prevalence rates between male and female major depressive disorder patients: a resting-state fMRI study. Clin Neurophysiol. 2014;125: 2232-2239.

22. Guo W, Song Y, Liu F, et al. Dissociation of functional and anatomical brain abnormalities in unaffected siblings of schizophrenia patients. Clin Neurophysiol. 2015;126:927-932.

23. Nofzinger EA, Buysse DJ, Germain A, Price JC, Miewald JM, Kupfer DJ. Functional neuroimaging evidence for hyperarousal in insomnia. Am J Psychiatry. 2004;161:2126-2128.

24. Van Dort CJ. Locus coeruleus neural fatigue: a potential mechanism for cognitive impairment during sleep deprivation. Sleep. 2016;39: $11-12$.

25. Bellesi M, Tononi G, Cirelli C, Serra PA. Region-specific dissociation between cortical noradrenaline levels and the sleep/wake cycle. Sleep. 2016;39:143-154. 
26. Thomas M, Sing H, Belenky G, et al. Neural basis of alertness and cognitive performance impairments during sleepiness. I. Effects of $24 \mathrm{~h}$ of sleep deprivation on waking human regional brain activity. $J$ Sleep Res. 2000;9:335-352.

27. Bonnet MH, Arand DL. Hyperarousal and insomnia. Sleep Med Rev. 1997;1:97-108.

28. Bonnet MH, Arand DL. Hyperarousal and insomnia: state of the science. Sleep Med Rev. 2010;14:9-15.

29. Chao-Gan Y, Yu-Feng Z. DPARSF: a Matlab toolbox for "pipeline" data analysis of resting-state fMRI. Front Syst Neurosci. 2010;4:13.

30. Joo EY, Noh HJ, Kim JS, et al. Brain gray matter deficits in patients with chronic primary insomnia. Sleep. 2013;36:999-1007.

31. Sexton CE, Storsve AB, Walhovd KB, Johansen-Berg H, Fjell AM. Poor sleep quality is associated with increased cortical atrophy in community-dwelling adults. Neurology. 2014;83:967-973.
32. Raichle ME, MacLeod AM, Snyder AZ, Powers WJ, Gusnard DA, Shulman GL. A default mode of brain function. Proc Natl Acad Sci USA. 2001;98:676-682.

33. Benington JH, Heller HC. Restoration of brain energy metabolism as the function of sleep. Prog Neurobiol. 1995;45:347-360.

34. Brooks VB. Cerebellar functions in motor control. Hum Neurobiol. 1984;2:251-260.

35. Cabeza R, Nyberg L. Imaging cognition II: an empirical review of 275 PET and fMRI studies. J Cogn Neurosci. 2000;12:1-47.

36. Gao JH, Parsons LM, Bower JM, Xiong J, Li J, Fox PT. Cerebellum implicated in sensory acquisition and discrimination rather than motor control. Science. 1996;272:545-547.

37. DelRosso LM, Hoque R. The cerebellum and sleep. Neurol Clin. 2014; 32:893-900.

\section{Publish your work in this journal}

Neuropsychiatric Disease and Treatment is an international, peerreviewed journal of clinical therapeutics and pharmacology focusing on concise rapid reporting of clinical or pre-clinical studies on a range of neuropsychiatric and neurological disorders. This journal is indexed on PubMed Central, the 'PsycINFO' database and CAS, and is the official journal of The International Neuropsychiatric Association (INA). The manuscript management system is completely online and includes a very quick and fair peer-review system, which is all easy to use. Visit http://www.dovepress.com/testimonials.php to read real quotes from published authors. 